Note: A replica liquid theory of binary mixtures

\title{
Note: A replica liquid theory of binary mixtures
}

\author{
Harukuni Ikeda, ${ }^{1}$, a) Kunimasa Miyazaki, ${ }^{1}$ and Atsushi Ikeda, b) \\ 1) Department of Physics, Nagoya University, Nagoya, 464-8602, Japan \\ ${ }^{2)}$ Graduate School of Arts and Sciences, University of Tokyo, Tokyo, 3-8-1, Japan
}

(Dated: 4 September 2018)

PACS numbers: 64.70.Q-, 05.20.-y, 64.70.Pf

The replica liquid theory (RLT) is a mean-field thermodynamic theory of the glass transition of supercooled liquids ${ }^{1}$. The theory was first developed for onecomponent monatomic systems. The RLT enables one to predict the ideal glass transition temperature, $T_{K}$, from a first-principles calculation, by considering the $m$ replicas of the original system 1 . Thermodynamic properties near $T_{K}$ are deduced by computing the free energy of a liquid consisting of $m$-atomic replica molecules and then taking the limit of $m \rightarrow 1$ at the end of the calculation. The RLT was later extended to binary systems $\underline{2} \underline{\underline{3}}$. However, it has been known that the binary RLT is inconsistent with its one-component counterpart; In the limit that all atoms are identical, the configurational entropy, $S_{c}$, and thus $T_{K}$ calculated by the binary RLT differ from those obtained by the one-component RLT $2, \underline{2}$. More specifically, an extra composition-dependent term, or the mixing entropy, remains finite in $S_{c}$ computed by the binary RLT. As discussed by Coluzzi et. al $\stackrel{2}{2}$, this contradiction originates from the assumption that each replica molecule consists of $m$-atoms of the same species. Physically, this is tantamount to assume that a permutation of atoms of one species with atoms of the other species in a given glass configuration is forbidden 2 . This is indeed the case if, say, the atomic radii of the two species are very different. Clearly this assumption is inappropriate if the two species are very similar or exactly identical because a permutation of the atoms of different species are allowed.

In this Short Note, we reformulate the RLT in order to resolve this problem. We consider a binary liquid composed of $A$ and $B$ atoms. The important step is to rewrite the expression of the grand canonical partition function in a form discussed by Morita and Hiroike ${ }^{4}$ as

$Z=\sum_{N=0}^{\infty} \frac{1}{N !} \prod_{i=1}^{N} \sum_{\nu_{i} \in\{A, B\}} \int d \boldsymbol{x}_{i} \exp \left[-\beta V_{N}+\beta \sum_{i=1}^{N} \mu_{\nu_{i}}\right]$,

where $\beta$ is the inverse temperature, $N$ is the total number of atoms, $V_{N}$ is the total potential energy. $\boldsymbol{x}_{i}$, $\nu_{i} \in\{A, B\}$, and $\mu_{\nu_{i}}$ are the position, species, and chemical potential of $i$-th atoms, respectively. Eq. (1) is mathematically equivalent to the standard expression for $Z^{\underline{5}}$.

\footnotetext{
a) Electronic mail: ikeda@r.phys.nagoya-u.ac.jp

b) Electronic mail: atsushi.ikeda@phys.c.u-tokyo.ac.jp
}

This expression can be readily generalized to the replicated liquid consisting of $m$-atomic replica molecules as

$$
\begin{aligned}
Z_{m} & =\sum_{N=0}^{\infty} \frac{1}{N !} \prod_{i=1}^{N}\left(\prod_{a=1}^{m} \sum_{\nu_{i}^{a} \in\{A, B\}} \int d \boldsymbol{x}_{i}^{a}\right) \\
& \times \exp \left[-\beta \sum_{a=1}^{m} V_{N}^{a}+\beta \sum_{i=1}^{N} \psi_{\bar{\nu}_{i}}\left(\overline{\boldsymbol{x}}_{i}\right)\right],
\end{aligned}
$$

where $\psi_{\bar{\nu}_{i}}\left(\overline{\boldsymbol{x}}_{i}\right) \equiv \sum_{a=1}^{m} \mu_{\nu_{i}^{a}}-u_{\bar{\nu}_{i}}\left(\overline{\boldsymbol{x}}_{i}\right)$ is the generalized chemical potential in which the external potential $u_{\overline{\nu_{i}}}\left(\overline{\boldsymbol{x}}_{i}\right)$ is included. $\overline{\boldsymbol{x}}_{i} \equiv\left(\boldsymbol{x}_{i}^{1}, \cdots, \boldsymbol{x}_{i}^{m}\right)$ and $\bar{\nu}_{i} \equiv\left(\nu_{i}^{1}, \cdots, \nu_{i}^{m}\right)$ denote the set of the positions and components of $m$ atoms of the $i$-th molecule. The advantage to express $Z_{m}$ à la Morita-Hiroike as Eq. (2) is that assigning a label of the component $\bar{\nu}_{i}$ to each atom enables one to describe replica molecules consisting of different set of species. The density field conjugated to $\psi_{\bar{\nu}}(\bar{x})$ can be written as

$$
\rho_{\bar{\nu}}(\overline{\boldsymbol{x}})=\sum_{i=1}^{N}\left\langle\prod_{a=1}^{m} \delta\left(\boldsymbol{x}^{a}-\boldsymbol{x}_{i}^{a}\right) \delta_{\nu_{i}^{a}, \nu_{i}^{a}}\right\rangle=\frac{\delta \log Z_{m}}{\delta \psi_{\bar{\nu}}(\overline{\boldsymbol{x}})} .
$$

Following the standard procedure $e^{5}$, one can express the free energy, $-\beta F$, by the Legendre transformation from $\psi_{\bar{\nu}}(\overline{\boldsymbol{x}})$ to $\rho_{\bar{\nu}}(\overline{\boldsymbol{x}})$, which can be written as the sum of the ideal and the excess parts;

$$
\beta F\left[\rho_{\bar{\nu}}(\overline{\boldsymbol{x}})\right]=-\sum_{\bar{\nu}} \int d \overline{\boldsymbol{x}} \rho_{\bar{\nu}}(\overline{\boldsymbol{x}})\left(1-\log \rho_{\bar{\nu}}(\overline{\boldsymbol{x}})\right)+\beta F_{\mathrm{ex}}\left[\rho_{\bar{\nu}}(\overline{\boldsymbol{x}})\right]
$$

The equilibrium free energy is obtained by minimizing Eq. (4) with respect to the density profile $\rho_{\bar{\nu}}(\overline{\boldsymbol{x}})$. For one-component systems, the standard procedure is to assume that $\rho_{\bar{\nu}}(\overline{\boldsymbol{x}})$ is Gaussian-shaped and use its width, or the cage size, as the minimization parameter $\underline{\underline{1}}$. Once the equilibrium free energy is obtained, $S_{c}$ is calculated by $S_{c}=\lim _{m \rightarrow 1} m^{2} \frac{\partial}{\partial m} \frac{\beta F}{m N}$. The ideal glass transition temperature, $T_{K}$, is identified as the point at which $S_{c}$ vanishes $\stackrel{1}{-}$. For binary liquids, however, the full computation is a challenging task because the cage sizes vary depending on the components $\bar{\nu}$. But, at least, one can demonstrate that the one-component result is correctly derived in the limit that atoms of two components are identical or very similar. In this limit, the density profile 
in the replica space can be written as

$$
\rho_{\bar{\nu}}(\overline{\boldsymbol{x}})=\rho \int d \boldsymbol{X} \prod_{a=1}^{m}\left(\sum_{\mu} c_{\mu} \delta_{\mu \nu^{a}} \gamma_{\Delta_{\mu}}\left(\boldsymbol{x}^{a}-\boldsymbol{X}\right)\right),
$$

where $\gamma_{A}(\boldsymbol{x}-\boldsymbol{X})=e^{-|\boldsymbol{x}-\boldsymbol{X}|^{2} / 2 \Delta} /(4 \pi \Delta)^{d / 2}$ is the Gaussian function centered at a reference position $\boldsymbol{X}$ with the cage size $\sqrt{\Delta} . \rho=N / V$ is the number density, and $c_{\nu}=N_{\nu} / N$ is the number fraction of the $\nu \in\{A, B\}$ species. Eq. (5) expresses that atoms of different species constitute a single replica molecules with the composition ratio of $c_{A}: c_{B}$. This ansatz corresponds to the limit where a permutation of the atoms of different species are allowed in a given glass configuration. The difference of the free energy from that of the one-component system $F_{1}$ is expressed by the difference in the ideal gas part (since the excess parts are identical in this limit) as

$$
\begin{aligned}
\beta \Delta F & \equiv \beta\left\{F\left[\rho_{\bar{\nu}}\right]-F_{1}[\rho]\right\} \\
& =-\sum_{\bar{\nu}} \int d \overline{\boldsymbol{x}} \rho_{\bar{\nu}}(\overline{\boldsymbol{x}})\left(1-\log \rho_{\bar{\nu}}(\overline{\boldsymbol{x}})\right) \\
& +\int d \overline{\boldsymbol{x}} \rho(\overline{\boldsymbol{x}})(1-\log \rho(\overline{\boldsymbol{x}})),
\end{aligned}
$$

where $\rho(\overline{\boldsymbol{x}})$ is the density profile of the one-component system. Because $\rho_{\bar{\nu}}(\overline{\boldsymbol{x}})=\rho(\overline{\boldsymbol{x}}) \times\left(\prod_{a} c_{\nu^{a}}\right)$ in the onecomponent limit, we arrive at $\beta \Delta F=m N \sum_{\mu} c_{\mu} \log c_{\mu}$. This implies that the $S_{c}$ of the binary RLT correctly converges to that of the one-component $S_{c, 1}$, because $\Delta S_{c} \equiv S_{c}-S_{c, 1}=m^{2} \partial_{m}(\beta \Delta F / m N)=0$.

In the opposite limit where the atoms of the two species are very different and one replica molecule consists of atoms solely of the one species, one can show that the previous results of binary $\mathrm{RLT}^{3}$ are recovered. In this case, the density profile should be written as

$$
\rho_{\bar{\nu}}^{\prime}(\overline{\boldsymbol{x}})=\rho \int d \boldsymbol{X} \sum_{\mu} c_{\mu}\left(\prod_{a=1}^{m} \delta_{\mu \nu^{a}} \gamma_{\Delta_{\mu}}\left(\boldsymbol{x}^{a}-\boldsymbol{X}\right)\right)
$$

Note the difference from Eq. (5); The order of the product over the atoms $a$ and the summation over the species $\mu$ have been exchanged. Due to the factor $\left(\prod_{a} \delta_{\mu \nu^{a}}\right)$ in Eq. (7), $\rho_{\bar{\nu}}^{\prime}(\overline{\boldsymbol{x}})$ vanishes unless each molecule consists of a single species. The non-vanishing component of the density profile is $c_{\nu} \rho \int d \boldsymbol{X} \prod_{a} \gamma_{\Delta_{\nu}}\left(\boldsymbol{x}^{a}-\boldsymbol{X}\right)(\nu=A$ or $B)$, which is exactly the density field employed in previous studies of the binary RLT 3 . Eq. (7) should not be used in the one-component limit because it gives a solution which is less stable than Eq. (5): Substituting Eq. (7) into Eq. (4) and optimizing the parameter $\Delta_{\nu}$, one finds $\beta \Delta F=N \sum_{\mu} c_{\mu} \log c_{\mu}$, which is larger than $\beta \Delta F$ from Eq. (5) 6 . This solution also leads to a pathological result $\Delta S_{c}=-\sum_{\mu} c_{\mu} \log c_{\mu}>0$; that is, $S_{c}$ calculated assuming Eq. (7) is larger than the correct one-component configurational entropy by the mixing entropy ${ }^{8}$.

In summary, we reformulate the RLT of binary, or multi-component mixtures, which correctly accounts for a permutation of the atoms in a glass configuration and show that it resolves the inconsistency between the onecomponent RLT and the binary RLT. The binary RLT in the previous studies is valid only in the limit where the atoms of different species are so different that a permutation of the atoms of different components is forbidden. In the one-component limit, one has to consider all possible permutations of atoms and adopts the density profile expressed as Eq. (5) to obtain the correct configurational entropy. For general cases between these two extreme limits, the density profile should be determined so as to minimize the free energy, Eq. (4). Its implementation, however, may be challenging. The results discussed above imply a possibility, for example, that a binary liquid of small and large hard spheres undergoes the glass-glass transition from the "disordered" glass where the both types of spheres constitute a replica molecule to the "ordered" glass where one replica molecule consists solely of one of the component, as the size ratio between the two components is varied, much the same way as the metallic alloys undergo the order-disorder phase transition as the interactions are varied 7 . Note that this possibility has also been pointed out in Ref $\stackrel{8}{*}$ in the context of the numerical simulation of the multi-component mixtures. Finally, we note that the generalization to $n$ component system is straightforward by allowing $\nu_{i}$ in Eq. (1) to take $n$-different states. Polydisperse case can be obtained in the $n \rightarrow \infty$ limit with a caveat about a subtlety to take the continuous limit (see Ref. [8])

\section{ACKNOWLEDGMENTS}

We are grateful to H. Yoshino, K. Hukushima, M. Ozawa, L. Berthier and F. Zamponi for helpful discussions. H. I. and K. M acknowledge JSPS KAKENHI Grants Number JP16H04034, JP25103005, JP25000002, H. I. was supported by Program for Leading Graduate Schools "Integrative Graduate Education and Research in Green Natural Sciences", MEXT, Japan.

${ }^{1}$ G. Parisi and F. Zamponi, "Mean-field theory of hard sphere glasses and jamming," Rev. Mod. Phys. 82, 789-845 (2010)

${ }^{2}$ B. Coluzzi, M. Mézard, G. Parisi, and P. Verrocchio, "Thermodynamics of binary mixture glasses," J. Chem. Phys. 111, 9039-9052 (1999).

${ }^{3}$ I. Biazzo, F. Caltagirone, G. Parisi, and F. Zamponi, "Theory of amorphous packings of binary mixtures of hard spheres," Phys. Rev. Lett. 102, 195701 (2009)

${ }^{4} \mathrm{~T}$. Morita and K. Hiroike, "A new approach to the theory of classical fluids. iii general treatment of classical systems," Progr. Theoret. Phys. 25, 537-578 (1961).

${ }^{5}$ J.-P. Hansen and I. R. McDonald, Theory of simple liquids (Elsevier, 1990).

${ }^{6}$ Exchange "larger" with "smaller" for $m<1$, see Ref 1 .

${ }^{7} \mathrm{~W}$. Greiner, L. Neise, and H. Stöcker, Thermodynamics and statistical mechanics (Springer Science \& Business Media, 2012).

${ }^{8} \mathrm{M}$. Ozawa and L. Berthier, "Does the configurational entropy of polydisperse particles exist?" arXiv preprint arXiv:1609.07979 (2016). 